University of Wollongong

Research Online

Faculty of Engineering - Papers (Archive)

Faculty of Engineering and Information

Sciences

2008

\title{
Magnetotransport and magnetic properties of weak ferromagnetic semiconductors: Ca doped LaCrO3
}

\author{
Gustavo A. Alvarez \\ University of Wollongong, gustavo@uow.edu.au \\ Xiaolin Wang \\ University of Wollongong, xiaolin@uow.edu.au \\ Germanas Peleckis \\ University of Wollongong, peleckis@uow.edu.au
}

S. X. Dou

University of Wollongong, shi@uow.edu.au

Jian G. Zhu

University of Technology, Sydney

See next page for additional authors

Follow this and additional works at: https://ro.uow.edu.au/engpapers

Part of the Engineering Commons

https://ro.uow.edu.au/engpapers/4234

\section{Recommended Citation}

Alvarez, Gustavo A.; Wang, Xiaolin; Peleckis, Germanas; Dou, S. X.; Zhu, Jian G.; and Lin, Zhi W.:

Magnetotransport and magnetic properties of weak ferromagnetic semiconductors: Ca doped LaCrO3 2008, 07B916-1-07B916-3.

https://ro.uow.edu.au/engpapers/4234

Research Online is the open access institutional repository for the University of Wollongong. For further information contact the UOW Library: research-pubs@uow.edu.au 


\section{Authors}

Gustavo A. Alvarez, Xiaolin Wang, Germanas Peleckis, S. X. Dou, Jian G. Zhu, and Zhi W. Lin 


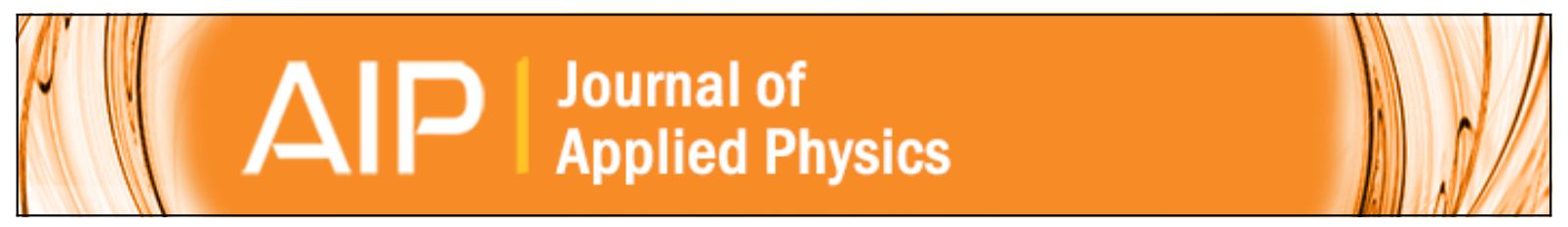

Magnetotransport and magnetic properties of weak ferromagnetic semiconductors: $\mathrm{Ca}$ doped La CrO 3

G. A. Alvarez, X. L. Wang, G. Peleckis, S. X. Dou, J. G. Zhu, and Z. W. Lin

Citation: Journal of Applied Physics 103, $07 \mathrm{~B} 916$ (2008); doi: 10.1063/1.2837624

View online: http://dx.doi.org/10.1063/1.2837624

View Table of Contents: http://scitation.aip.org/content/aip/journal/jap/103/7?ver=pdfcov

Published by the AIP Publishing

\section{Articles you may be interested in}

High temperature thermoelectric characteristics of $\mathrm{Ca} 0.9 \mathrm{R} 0.1 \mathrm{MnO} 3(\mathrm{R}=\mathrm{La}, \mathrm{Pr}, \ldots, \mathrm{Yb})$

J. Appl. Phys. 104, 093703 (2008); 10.1063/1.3003065

Magnetic and electrical transport properties of La $0.67 \mathrm{Ca} 0.33 \mathrm{MnO} 3$ (LCMO): x SiCN composites J. Appl. Phys. 95, 7106 (2004); 10.1063/1.1667447

Transport, magnetic, specific heat, internal friction, and shear modulus in the charge ordered La $0.25 \mathrm{Ca} 0.75$ MnO 3 manganite

J. Appl. Phys. 94, 514 (2003); 10.1063/1.1583150

Spin hopping in a discontinuous La 0.7 Ca $0.3 \mathrm{MnO} 3$ film

Appl. Phys. Lett. 80, 2144 (2002); 10.1063/1.1462870

Electrical and magnetic phases of the layered perovskite Ca 4x La x Mn 3010

Appl. Phys. Lett. 72, 853 (1998); 10.1063/1.120915

\section{A|P| Journal of}

Journal of Applied Physics is pleased to announce André Anders as its new Editor-in-Chief 


\title{
Magnetotransport and magnetic properties of weak ferromagnetic semiconductors: $\mathrm{Ca}$ doped $\mathrm{LaCrO}_{3}$
}

\author{
G. A. Alvarez, X. L. Wang, ${ }^{\text {a) }}$ G. Peleckis, and S. X. Dou \\ Spintronic and Electronic Materials Group, Institute for Superconducting and Electronic Materials, \\ University of Wollongong, Wollongong, New South Wales 2522, Australia \\ J. G. Zhu and Z. W. Lin \\ Faculty of Engineering, University of Technology Sydney, Sydney, New South Wales 2007, Australia
}

(Presented on 7 November 2007; received 11 September 2007; accepted 14 November 2007; published online 26 March 2008)

\begin{abstract}
We report the magnetic and magnetotransport properties of $\mathrm{Ca}$ doped $\mathrm{La}_{1-x} \mathrm{Ca}_{x} \mathrm{CrO}_{3}(x=0,0.1,0.2$, and 0.3). Both the magnetic and transport properties are very sensitive to Ca substitution. The Néel temperature $T_{N}$ decreases substantially with increasing calcium doping concentration from 290 to $160 \mathrm{~K}$. A weak ferromagnetic state with large coercive fields of up to $3 \mathrm{~T}$ is present for the $\mathrm{Ca}$ doped $\mathrm{LaCrO}_{3}$. The temperature dependence of the resistivity shows that all the $\mathrm{Ca}$ doped compounds are semiconducting and their resistivities decrease with increasing Ca for low doping levels. The resistivity curves show thermally activated behavior and a variable range hopping behavior at high temperatures. The magnetotransport measurements show a negative magnetoresistance. Furthermore, an anomalous peak was observed in the field dependence of magnetoresistance for the LCCO materials. () 2008 American Institute of Physics.
\end{abstract}

[DOI: $10.1063 / 1.2837624]$

\section{INTRODUCTION}

The manganese oxides have been studied extensively because of the strong coupling among the charge, lattice, and spin degrees of freedom that leads to colossal magnetoresistance. ${ }^{1}$ Similar behavior has been observed in some cobalt oxides which exhibit a well-known low-spin to high-spin transition. ${ }^{2}$ The electrical, magnetic, and structural properties of $\mathrm{Cr}$ oxides have been reported in a number of publications. $\mathrm{LaCrO}_{3}$ is antiferromagnetic ${ }^{3,4}$ below $290 \mathrm{~K}$. It is a poor electrical conductor at room temperature, but its high conductivity at high temperature as well as its ability to withstand high temperatures has made it useful as an interconnect between fuel cells and as a material for heating elements. ${ }^{4}$ A limited number of doping studies of $\mathrm{LaCrO}_{3}$, focusing on the electrical and magnetic properties, have been conducted whereby divalent ions such as $\mathrm{Sr}$ or $\mathrm{Ca}$ are substituted for trivalent La. Sakai et al. $^{3}$ investigated doping levels up to $75 \% \mathrm{Ca}$ and a number of oxygen concentrations. They reported the crystal structure over a wide range of temperatures $12<T<1300 \mathrm{~K}$, revealing an orthorhombic to rhombohedral phase transition at about $600 \mathrm{~K}$ for $\mathrm{La}_{0.80} \mathrm{Ca}_{0.20} \mathrm{CrO}_{3}$. In addition, they reported the antiferromagnetic structure as $G$ type for a number of $\mathrm{Ca}$ concentrations and conducted some magnetic susceptibility measurements. Tezuka et al. ${ }^{4}$ published an investigation of the magnetic and structural properties of $\mathrm{La}_{1-x} \mathrm{Sr}_{x} \mathrm{CrO}_{3}$ with 0 $<x<0.25$. They also found an orthorhombic to rhombohedral phase transition for $0 \%$ and 5\% Sr doping at 526 and $413 \mathrm{~K}$, respectively. Furthermore, they were able to establish the antiferromagnetic structure associated with both of these

\footnotetext{
${ }^{a)}$ Author to whom correspondence should be addressed. Electronic mail: xiaolin@uow.edu.au.
}

phases for $x=0.15$ as $G$ type. The dc electrical transport of $\mathrm{La}_{1-x} \mathrm{Sr}_{x} \mathrm{CrO}_{3}(0<x<0.40)$ is thermally activated in the range $300<T<2000 \mathrm{~K}$ with activation energies between 0.11 and $0.19 \mathrm{eV}$ and temperature-dependent behavior consistent with conduction by small polarons. ${ }^{5,6}$

$\mathrm{LaCrO}_{3}$ is an interesting compound with transition metal ions that have three electrons in the $3 d$ shell, which leads to total spin $S=\frac{3}{2}$. The antiferromagnetic structure is $G$ type, whereby each $\mathrm{Cr}^{3+}$ ion is antiferromagnetically coupled to its neighbor. This structure is predicted by considerations of covalency by Goodenough, ${ }^{7}$ arguments which should be valid for the $\mathrm{Cr}$ transition metal compounds. Structural transitions, such as the orthorhombic to rhombohedral phase transition, were observed ${ }^{3,4}$ in $\mathrm{La}_{0.80} \mathrm{Ca}_{0.20} \mathrm{CrO}_{3}$.

In the present paper, the structural, magnetic, and electrical properties of $\mathrm{La}_{1-x} \mathrm{Ca}_{x} \mathrm{CrO}_{3}$ are investigated. The main goal of this paper is to provide more information about the influence of doping on the antiferromagnetism and weak ferromagnetic moment. Detailed measurements of the magnetic properties reveal that the weak ferromagnetic moment is likely due to canting of the antiferromagnetically ordered $\mathrm{Cr}$ moments.

\section{EXPERIMENTAL}

The polycrystalline samples of $\mathrm{La}_{1-x} \mathrm{Ca}_{x} \mathrm{CrO}_{3}(x=0,0.1$, 0.2 , and 0.3 ) were synthesized using standard solid-state reaction. Stoichiometric quantities of $\mathrm{La}_{2} \mathrm{O}_{3}, \mathrm{CaCO}_{3}$, and $\mathrm{Cr}_{2} \mathrm{O}_{3}$ were weighed and mixed in a mortar followed by reaction in an alumina crucible at $1200{ }^{\circ} \mathrm{C}$ overnight. The samples were then reground, pressed into pellets, and reacted at $1450{ }^{\circ} \mathrm{C}$ for $48 \mathrm{~h}$ followed by slow cooling in air at a rate of $1.5^{\circ} \mathrm{C} / \mathrm{min}$. All samples were investigated with powder $\mathrm{x}$-ray diffraction and found to exhibit only the perovskite 

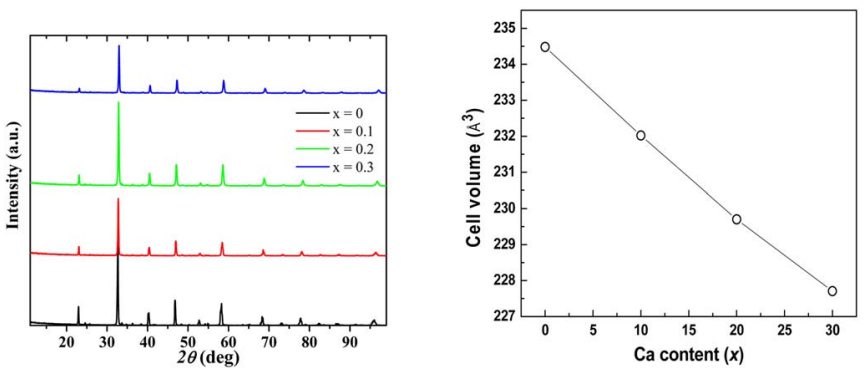

FIG. 1. (Color online) (a) X-ray diffraction patterns and (b) unit cell volume as determined from Rietveld refinement of powder $\mathrm{x}$-ray diffraction data for $\mathrm{La}_{1-x} \mathrm{Ca}_{x} \mathrm{CrO}_{3}$ samples.

phase typical to these compounds and no secondary impurity phases. Measurements were conducted using a Quantum Design physical properties measurement system. In measuring the magnetization $M$ versus $T$, samples are cooled in a field from $350 \mathrm{~K}$ at a rate of $1 \mathrm{~K} /$ min under magnetic fields up to $8 \mathrm{~T}$. Data are acquired in the temperature sweep mode. The magnetoresistance and electrical resistivity were measured using a four-probe de technique.

\section{RESULTS AND DISCUSSION}

Phase purity of the as prepared $\mathrm{La}_{1-x} \mathrm{Ca}_{x} \mathrm{CrO}_{3}$ samples was analyzed utilizing $\mathrm{x}$-ray diffraction and Rietveld refinement. The x-ray diffraction patterns for these samples are shown in Fig. 1(a). As we can see, samples are of characteristic orthorhombic $\mathrm{LaCrO}_{3}$ phase with minute amounts of unreacted $\mathrm{Cr}_{2} \mathrm{O}_{3}$ impurities. Rietveld refinement analysis revealed that the amount of $\mathrm{Cr}_{2} \mathrm{O}_{3}$ impurity for the samples was below $1 \%$. Ca substitution for La caused shrinkage of all three lattice parameters, and correspondingly unit cell volume [Fig. 1(b)]. These results are in a good agreement with previously published data, ${ }^{3}$ where similar decrease in unit cell volume was obtained for oxidized and reduced $\mathrm{Ca}-$ substituted $\mathrm{LaCrO}_{3}$ samples. In an octahedral coordination $r_{\left(\mathrm{Ca}^{2+}\right)}=1.12 \AA$ and $r_{\left(\mathrm{La}^{3+}\right)}=1.16 \AA$, respectively. Thus, the observed decrease in unit cell volume with increasing $\mathrm{Ca}$ content in the samples is reasonable. Furthermore, Rietveld analysis revealed that with increasing Ca content, site occupancy for oxygen ions in the structure decreases. This might be caused by the charge imbalance, as triply charged $\mathrm{La}^{3+}$ ions are replaced by doubly charged $\mathrm{Ca}^{2+}$ ions. Similarly, Sakai et $a l^{3}{ }^{3}$ showed that in La rich samples, the created oxygen vacancies are confined to small $\mathrm{Ca}^{2+}-V_{\mathrm{O}}-\mathrm{Ca}^{2+}$ clusters. In addition, the increase in $\mathrm{Ca}$ content causes a shift in the arrangement of $\mathrm{CrO}_{6}$ octahedrons in the structure. The $\mathrm{Cr}-\mathrm{O}-\mathrm{Cr}$ angles in the $\mathrm{CrO}_{2}$ planes become larger with increasing $\mathrm{Ca}$ content.

The magnetization $M$ versus temperature $T$ in an applied magnetic field $H=2000$ Oe is plotted in Fig. 2 for selected specimens. These data were collected by cooling the samples in the constant magnetic field from $350 \mathrm{~K}$. Ca doping depresses the magnetic transition $T_{N}$ in a manner that is more severe than that observed for $\mathrm{Sr}$ doping. ${ }^{4}$ This can be understood through structural considerations. It is important to note that the average $\mathrm{Cr}-\mathrm{O}-\mathrm{Cr}$ bond angle is about $160^{\circ}$, according to powder neutron diffraction data on $\mathrm{LaCrO}_{3}$;

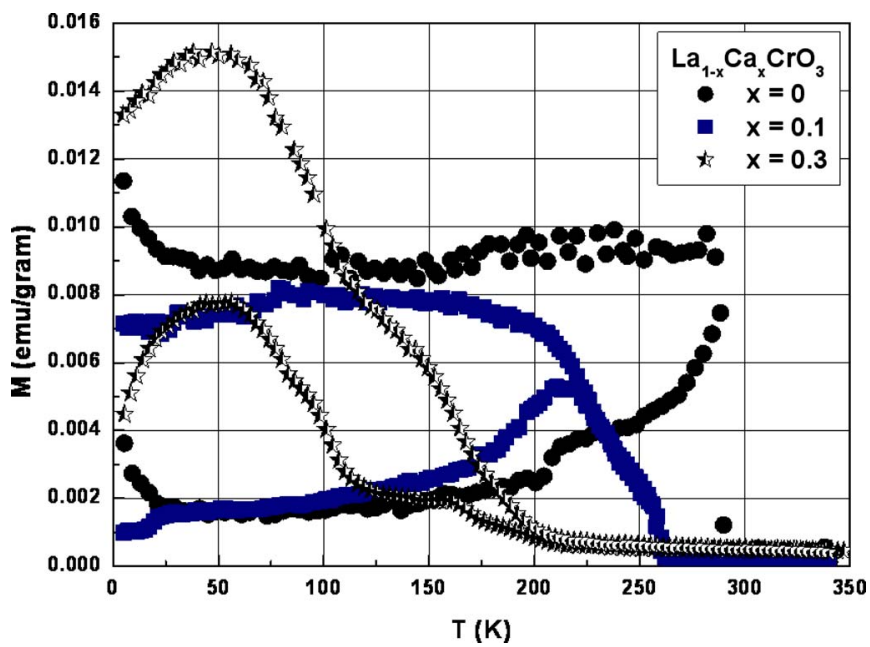

FIG. 2. (Color online) Magnetization vs temperature at 2000 Oe for $\mathrm{La}_{1-x} \mathrm{Ca}_{x} \mathrm{CrO}_{3}$ samples with $x=0,0.10$, and 0.30 .

also, a 30\% substitution of $\mathrm{Ca}$ for La increases the angle by only ${ }^{3}$ about $1^{\circ}$. As a result, since Ca substitution has a rather small effect on the bond angle important for magnetic exchange, it is highly possible that the majority of the herein observed depression of $T_{N}$ results from the doped-hole concentration which increases with $x$. Thus, the $\mathrm{La}_{1-x} \mathrm{Ca}_{x} \mathrm{CrO}_{3}$ system provides a better measure of the influence of the $d$-orbital electron vacancies on $T_{N}$.

Doping $\mathrm{Ca}$ into $\mathrm{LaCrO}_{3}$ enhances the magnitude of $M$ below the magnetic transition and has a strong effect on the temperature dependence of $M$ in the antiferromagnetic state. We investigated the behavior of $M$ on $H$ in some detail in order to better understand this effect. Some example data are illustrated in Fig. 3 for $\mathrm{La}_{1-x} \mathrm{Ca}_{x} \mathrm{CrO}_{3}$ samples where $M$ versus $H$ is shown at different temperatures above and below $T_{N}$. We observe in Fig. 3 extremely large coercive fields for this system. For example, the coercive field at $10 \mathrm{~K}$ is about $3.5 \mathrm{~T}$ for $x=0.1$ and $3 \mathrm{~T}$ for $x=0.20$. The observation of large coercive fields reveals that a substantial magnetic field is required to orient the magnetic domains along the direction of the applied field. The large coercive field may be associated with the microstructure of the specimens. ${ }^{8}$ It would probably not be present if the ferromagnetic component were due to impurities. Thus, we believe the observed

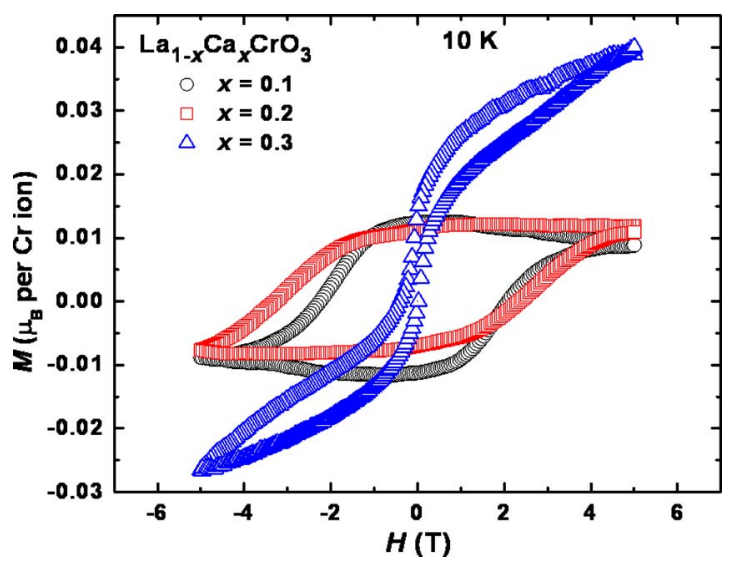

FIG. 3. (Color online) Magnetization $M$ vs magnetic field $H$ at $10 \mathrm{~K}$ for $\mathrm{La}_{1-x} \mathrm{Ca}_{x} \mathrm{CrO}_{3}$ samples with $x=0.10,0.20$, and 0.30 . 

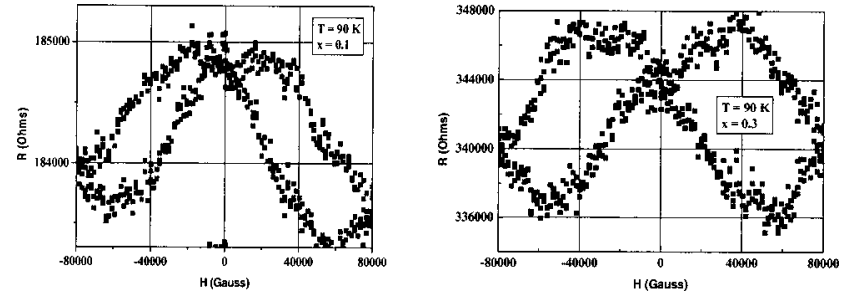

FIG. 4. Butterfly magnetoresistance of $\mathrm{La}_{1-x} \mathrm{Ca}_{x} \mathrm{CrO}_{3}$ samples measured at $90 \mathrm{~K}$, obtained on cycling the field from $+8 \mathrm{~T}$ to $-8 \mathrm{~T}$. (a) $x=0.1$, (b) $x$ $=0.3$.

ferromagnetic component to be intrinsic and the result of a minor amount of canting of the antiferromagnetic moments. Such canting or noncollinear ferromagnetism is common in perovskite compounds ${ }^{9,10}$ and has been reported ${ }^{3}$ from neutron powder diffraction for $\mathrm{La}(\mathrm{Ca}) \mathrm{CaO}_{3}$.

Manetoresistance measurements for $x=0.1$ and 0.3 (Fig. 4) show a butterfly-hysteresis curve at $90 \mathrm{~K}$ obtained on cycling the field from +8 to $-8 \mathrm{~T}$. This small ferromagnetic component shrinks in magnitude with increasing temperature, vanishing close to the Néel temperature. As a function of $x$, the ferromagnetic component increases rapidly at $x$ $=0.2$, followed by a decrease at $x=0.3$. One effect that may contribute to the observed magnetoresistance is the anisotropic magnetoresistance effect, commonly associated with the presence of substantial $d$-band splitting and spin-orbit coupling in transition metals and alloys. ${ }^{11}$ In this case, the magnetoresistance ratio is a function of the angle between the current and the magnetization of the samples and is dependent on the density of $d$-like states at the Fermi level, sample quality, temperature, etc. As the spins are gradually turned from their random local easy directions toward the directions of the applied field, this generates most of the hysteresis in the magnetoresistance, but little hysteresis in the magnetization. The hysteresis of the magneto resistance arises because there is some modification of the short range order among the transverse components as the spin configuration falls back from its aligned state with the removal of the applied field. These correlations may be antiferromagnetic in the virgin state, ${ }^{12}$ and then end to be ferromagnetic as saturation is approached, turning antiferromagnetic again when the field is reversed.

The data may suggest that weak localization is possible in the presence of magnetic scattering from magnetically concentrated impurities, ${ }^{13}$ however, it is highly possible that a different magnetization process operates at temperatures below $T_{N}$ for our LCCO samples.

The dc electrical resistivity $\rho$ was measured in the temperature range $100<T<350 \mathrm{~K}$. Example data for $x=0.1$, 0.2 , and 0.3 are presented in Fig. 5 as $\ln (\rho)$ versus $1000 / T$. No features are apparent in the region near $T_{N}$ for any of the samples, suggesting weak coupling between the charge carriers and the magnetism. It is interesting to note that $\rho$ $(300 \mathrm{~K})$ falls with $x$ until 0.20 , then a sharp increase is ob-

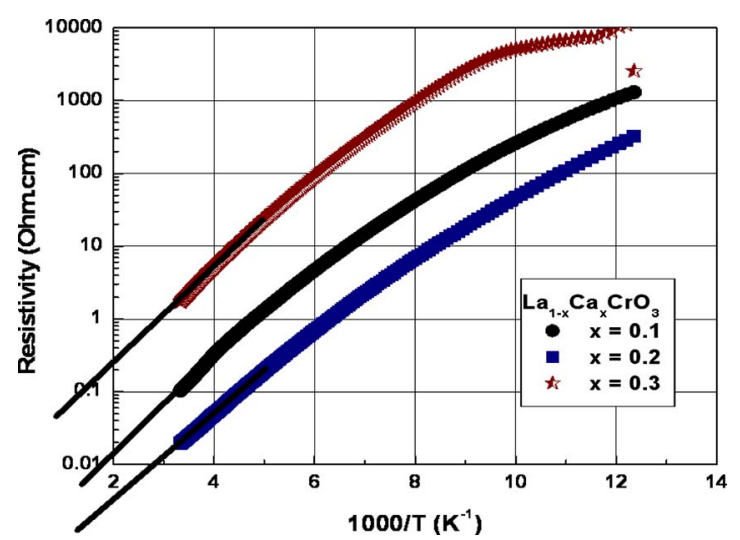

FIG. 5. (Color online) Electrical resistivity vs inverse temperature for $\mathrm{La}_{1-x} \mathrm{Ca}_{x} \mathrm{CrO}_{3}$ samples in zero magnetic field.

served at $x=0.30$. This value of $x$ coincides as well with a loss of the ferromagnetic moment, suggesting that the magnetism and electrical conduction both exhibit unusual behavior at $x=0.30$; such behavior could result from a change in the spin arrangement associated with the antiferromagnetic order and coupling between the charge carriers and magnetic moments. Doping-induced changes in the spin arrangement of the magnetic lattice are well known in systems such as $\mathrm{La}_{1-x} \mathrm{Ca}_{x} \mathrm{MnO}_{3}{ }^{14}$ To provide an energy gap $E_{g}$ that gives a measure of the thermal excitation needed for conduction, we fit the data to $\rho(T)=\rho \exp \left(E_{g} / k_{B} T\right)$. The data were fitted in the temperature range $200<T<500 \mathrm{~K}$; straight-line behavior was observed in this temperature range and the obtained energy gaps values are in the same range as those reported ${ }^{6}$ for $\mathrm{La}_{1-x} \mathrm{Sr}_{x} \mathrm{CrO}_{3}$. We believe that electrical resistivity data on single crystals or high-quality thin films are necessary to fully investigate the electrical conduction properties of this system. For this reason, the results presented herein are simply a guide to the general behavior and are certainly influenced by grain boundary effects due to the polycrystalline nature of the samples.

This work was supported by the funding from the Australian Research Council through ARC Discovery project.

${ }^{1}$ J. M. D. Coey, M. Viret, and S. von Molnár, Adv. Phys. 48, 167 (1999).

${ }^{2}$ J. Wu and C. Leighton, Phys. Rev. B 67, 174408 (2003).

${ }^{3}$ N. Sakai, H. Fjellvåg, and B. C. Hauback, J. Solid State Chem. 121, 202 (1996).

${ }^{4}$ K. Tezuka, Y. Hinatsu, A. Nakamura, T. Inami, Y. Shimojo, and Y. Morii, J. Solid State Chem. 141, 404 (1998).

${ }^{5}$ J. B. Webb, M. Sayer, and A. Mansingh, Can. J. Phys. 55, 1725 (1977).

${ }^{6}$ D. P. Karim and A. T. Aldred, Phys. Rev. B 20, 2255 (1979).

${ }^{7}$ J. B. Goodenough, Phys. Rev. 100, 564 (1955).

${ }^{8}$ R. M. Bozorth, Ferromagnetism (IEEE, New York, 1978).

${ }^{9}$ P.-G. de Gennes, Phys. Rev. 118, 141 (1960).

${ }^{10}$ J. J. Neumeier and J. L. Cohn, Phys. Rev. B 61, 14319 (2000).

${ }^{11}$ T. R. McGuire and R. I. Potter, IEEE Trans. Magn. 11, 1018 (1975).

${ }^{12}$ B. Boucher, A. Lienard, J. P. Rebouillat, and J. Schweizer, J. Phys. F: Met. Phys. 9, 1421 (1979); 9, 1433 (1979).

${ }^{13}$ J. B. Bieri, A. Fert, G. Creuzet, and A. Schuhl, J. Phys. F: Met. Phys. 16, 2099 (1986).

${ }^{14}$ E. O. Wollan and W. C. Koehler, Phys. Rev. 100, 545 (1955). 